\title{
Contact et frottement en dynamique des systèmes de corps rigides
}

\author{
Jean Jacques Moreau \\ Laboratoire de Mécanique et Génie Civil \\ Université Montpellier II/CNRS, UMR 5508 \\ Case 048, Université Montpellier II, 34095 Montpellier Cedex 5, France. \\ moreau@lmgc.univ-montp2.fr
}

\begin{abstract}
RÉSUMÉ. Une formulation de la dynamique de collections de corps rigides, prenant en compte le caractère unilatéral des liaisons d'impénétrabilité, le frottement sec en cas de contact et certaines lois de restitution en cas de collision est présentée. Elle débouche sur le calcul numérique de l'évolution par des d'algorithmes à temps discrétisé de type implicite.

ABSTRACT. A formulation of the dynamics of rigid body collections, taking into account the unilateral character of non-interpenetrability constraints, dry friction at possible contact points and certain restitution laws in the event of collisions is presented. It results in timestepping algorithms of the implicit type for calculating evolutions.

MOT-CLÉS : contact unilatéral, éléments discrets.

KEY WORDS : unilateral contact, discrete elements,.
\end{abstract}




\section{Introduction}

Le présent article fait suite à une conférence générale donnée au 4 e Colloque $\mathrm{Na}$ tional en Calcul des Structures, Giens, 18-21 mai 1999. Il reprend donc quelques éléments du texte inséré dans les Actes de ce Colloque [22], mais fournit davantage d'information sur l'implémentation des techniques numériques. On a aussi renoncé à certaines simplifications faites alors dans un but "pédagogique".

Dans des domaines d'application divers, le besoin apparait de calculer le mouvement, ou de discuter l'équilibre, de collection de corps rigides ou déformables entre lesquels des contacts, usuellement affectés de frottement, sont susceptibles de s'établir ou de se rompre. La réflexion théorique sur de tels problèmes de Dynamique en présence de liaisons unilatérales remonte à E. Delassus [7], dans le premier quart de ce siècle. Quant aux techniques numériques, il faut citer le travail de pionnier de P. Cundall [6], motivé par la géomécanique.

La situation peut être qualifiée de non régulière pour les raisons suivantes.

Les conditions géométriques de non-interpénétration des membres du système et, éventuellement, celles de leur confinement unilatéral par des parois extérieures s'expriment par une famille d'inégalités concernant les paramètres de position. Dans la variété $\mathcal{Q}$ de ces paramètres l'ensemble des positions permises, au lieu d'être une sous-variété régulière comme dans la Mécanique Analytique traditionnelle, est une région de $\mathcal{Q}$ dont la frontière est faite de morceaux d'hypersurfaces (des millions de tels morceaux dans les applications usuelles aux granulats) se rejoignant selon des "arêtes". Cela peut s'appeler la nonrégularité spatiale.

La réalisation mécanique des liaisons d'impénétrabilité implique des forces de contact, gouvernées par des lois très peu régulières. Par exemple dans le cas le plus simple, celui d'un contact ponctuel à frottement nul, la loi consiste en la relation suivante entre le vecteur force de contact $\mathbf{r}$ et l'interstice $g \geq 0$ : si $g>0$ on a $\mathbf{r}=0$ tandis que si $g=0, \mathbf{r}$ est indéterminé sur la demi-droite normale aux corps en contact. Par ailleurs, si un frottement sec est pris en compte, il introduit une relation entre la force de contact et la vitesse relative locale des corps concernés dont le graphe n'est pas une variété régulière, mais est fait de parties à première vue hétéroclites. Tout cela peut s'appeler la non régularité en loi. Noter que les relations en question ne permettent d'exprimer aucune des variables concernées comme fonction univoque des autres.

Enfin, si une collision survient entre des corps traités comme rigides, on attend des sauts de vitesse: c'est la non régularité temporelle.

Dans la majorité des techniques numériques employées à ce jour, ces difficultés sont abordées au moyen d'approximations régularisantes. L'impénétrabilité des corps est remplacée par des lois de répulsion suffisamment abruptes qui entrent en jeu lorsque deux d'entre eux s'approchent. De même, la loi de Coulomb est usuellement adoucie. On est ainsi ramené à des équations différentielles justiciables de techniques numériques classiques. Mais, dans chaque cas d'espèce, un compromis doit être accepté entre l'exigence de précision et la raideur des équations approximantes. Cette raideur impose des pas de 
discrétisation très petits et, souvent, des inerties ou des viscosités artificielles sont introduites pour assurer la stabilité numérique. L'application d'une telle stratégie de calcul à des situations proprement dynamiques demande beaucoup de précautions et de savoir-faire. Son application est moins problématique dans la recherche d'états d'équilibre ou le calcul d'évolutions quasi-statiques, indifférentes à ce que la dynamique invoquée pour passer d'un quasi-équilibre à un autre peut avoir d'artificiel.

Les techniques numériques basées sur la régularisation des liaisons d'impénétrabilité sont souvent désignées par le sigle $M D$ (pour Molecular Dynam$i c s$, en référence à leur usage dans les simulations numériques de dynamique moléculaire [29]).

Noter que, dans des logiciels de dynamique des machines, des approximations régularisantes sont communément appliquées aussi à des liaisons bilatérales, si les fonctionnalités du programme font introduire ces liaisons dans un système mécanique antérieurement paramétrisé [10].

Dans ce dernier domaine d'applications, le nombre d'objets concernés dépassant rarement une dizaine, une mise en équations exacte des problèmes à liaisons unilatérales reste également praticable. On cherchera à identifier des intervalles de temps sur lesquels l'ensemble des contacts effectifs demeure constant. Sur chacun de ces intervalles, le mouvement est calculé comme s'il s'agissait de liaisons bilatérales classiques, éventuellement avec frottement. On surveille les signe des composantes normales des réactions: si l'un d'entre eux devient incompatible avec l'unilatéralité, on conclut que le mouvement subséquent doit être calculé autrement. Delassus montra qu'en présence de plusieurs contacts, ceux qui cessent ne sont pas nécessairement ceux qui ont déclanché l'alerte et proposa un mode de détermination du nouveau statut des contacts n'exigeant pas l'essai de toutes les combinaisons. Aujourd'hui, les arguments de Delassus sont remplacés par ce qu'on appelle, en Analyse Non Régulière, des problèmes de complémentarité [13][23].

Le frottement sec, ou tout autre phénomène impliquant des lois à seuil, peut de la même façon faire apparaître des instants critiques.

Un autre signal exigeant que les équations écrites soient reconsidérées est l'apparition d'un nouveau contact, c'est-à-dire une collision. Le calcul du nouvel état de vitesse exige des informations phénoménologiques sur le processus de choc. Il est facile d'en concevoir sous la forme de relations entre les vitesses avant et après le choc, mais leur conformité à la réalité reste à discuter selon les circonstances [26].

L'approche décrite ci-dessus est communément désignée par le sigle $E D$ (pour Event Driven).

Le présent exposé vise à donner une idée d'une technique numérique plus récente [15][16] intitulée $C D$ (pour Contact Dynamics), basée elle aussi sur la mise en équations exacte, mais capable au stade actuel de traiter des systèmes d'une dizaine de milliers de solides. Elle permet de prendre en compte aussi la déformabilité des corps en présence [11][1]. Elle incorpore des procédures très simples pour traiter les collisions, mais la validité phénoménologique de 
ces procédures appelle naturellement les mêmes réserves que celle des autres formalisations.

En mécanique des granulats, des géomatériaux ou des maçonneries, les simulations numériques basées sur la prise en compte individuelle des grains ou des blocs, qu'elles soient de type $M D, E D$ ou $C D$, sont qualifiés de méthodes $D E M$ (pour Discrete Element Method ou Distinct Element Method), par opposition avec la stratégie FEM (Finite Element Method) utilisée lorsqu'une loi de comportement homogénisée a été choisie, assimilant le granulat ou la maçonnerie à un milieu continu.

\section{Exemple élémentaire : une particule ponctuelle en dimension 3}

\subsection{Confinement sans frottement: première approche}

Le cas particulier suivant fournit une introduction à la méthode $C D$.

Dans l'espace rapporté aux axes galiléens $O x_{1}, x_{2}, x_{3}$, une particule ponctuelle $Q$ de masse $m$, soumise à un champ de forces dépendant éventuellement du temps $(t, x) \mapsto X(t, x)$, est confinée dans la région $\Phi(t)=\left\{x \in \mathbf{R}^{3} \mid f(t, x) \leq 0\right\}$ par une paroi matérielle ayant un mouvement imposé, d'équation $f(t, x)=0$. L'inconnue principale de la dynamique est la fonction vitesse $t \mapsto u \in \mathbf{R}^{3}$, dont la fonction position $t \mapsto q$ se déduit par

$$
q(t)=q\left(t_{0}\right)+\int_{t_{0}}^{t} u(s) d s .
$$

Sur tout intervalle de temps où $u$ est suffisamment régulière (précisons : absolument continue), l'équation de la dynamique s'écrit

$$
m \frac{d u}{d t}=X(t, q(t))+r
$$

L'inconnue $r \in \mathbf{R}^{3}$ est la force de liaison, c'est-à-dire la réaction de la paroi en cas de contact. Dans cette Section, on la suppose régie par les conditions suivantes :

Le confinement dont l'effet géométrique est

$$
f(t, q) \leq 0
$$

est supposé réalisé par contact,

$$
f(t, q)<0 \Rightarrow r=0 .
$$

On admet de plus que le contact éventuel est sans frottement, i.e.

$$
f(t, q)=0 \quad \Rightarrow \quad \exists \lambda \in \mathbf{R}: r=\lambda \nabla f(t, q)
$$


(le gradient $\nabla f(t, q)$, vecteur de $\mathbf{R}^{3}$ normal à la paroi dans le sens sortant de $\Phi(t)$, sera supposé non nul). Enfin le contact est déclaré non adhésif,

$$
\lambda \leq 0
$$

Le système de conditions (3) à (6) constitue une relation, vérifiée à tout instant, entre les inconnues $q$ et $r$ et en laquelle se résume dans le cas présent la totalité des informations disponibles concernant la liaison invoquée. On va voir que ces conditions ne sont pas aussi hétéroclites qu'il paraît.

Pour tout $q$ appartenant à la surface frontière $f=0$, la demi-droite engendrée dans $\mathbf{R}^{3}$ par le vecteur $\nabla f(t, q)$ constitue le cône normal sortant de la région $\Phi(t)$ au point $q$, noté $\mathrm{N}_{\Phi(t)}(q)$. Il se révèle cohérent d'étendre comme suit la définition de ce cône pour tout $q$ dans $\mathbf{R}^{3}$ : on pose $\mathrm{N}_{\Phi(t)}(q)=\emptyset$ si $q \notin \Phi(t)$ et $\mathrm{N}_{\Phi(t)}(q)=\{0\}$ si $q$ est intérieur à $\Phi(t)$. Moyennant quoi on constate que le système de conditions $(3)$ à $(6)$ se condense en

$$
-r \in \mathrm{N}_{\Phi(t)}(q)
$$

L'objectif traditionnel de la Mécanique Analytique, à savoir l'élimination des réactions des liaisons sans frottement, est atteint ici en rapprochant (2) et (7): les mouvements recherchés sont les solutions de l'inclusion différentielle

$$
X(t, q(t))-m \frac{d u}{d t} \in \mathrm{N}_{\Phi(t)}(q(t)),
$$

plus précisément une inclusion intégro-différentielle, compte tenu de (1). Noter que cette écriture implique $q(t) \in \Phi(t)$, puisqu'autrement le second membre serait vide.

\subsection{Confinement sans frottement: seconde approche}

L'inclusion différentielle (8) est d'un traitement difficile, tant du point de vue analytique [4][12] (existence et unicité éventuelle des solutions, convergence des approximations, etc.) que numérique. L'essence de la méthode $C D$ est de lui substituer un équivalent plus traitable.

Pour un instant $t_{1}$ tel que $f\left(t_{1}, q\left(t_{1}\right)\right)<0$, la permanence de (3) n'impose rien à la vitesse $u\left(t_{1}\right)$. Mais si $f\left(t_{1}, q\left(t_{1}\right)\right)=0$, la vitesse à droite $u^{+}\left(t_{1}\right)$, supposée exister, vérifie nécessairement (formule des fonctions composées)

$$
f_{t}^{\prime}\left(t_{1}, q\left(t_{1}\right)\right)+u^{+}\left(t_{1}\right) \cdot \nabla f\left(t_{1}, q\left(t_{1}\right)\right) \leq 0
$$

et la vitesse à gauche $u^{-}\left(t_{1}\right)$ l'inégalité opposée. Quels que soient $t$ et $q$, même n'assurant pas (3), posons

$$
W(t, q):=\left\{\begin{array}{lc}
\left\{v \in \mathbf{R}^{3} \mid f_{t}^{\prime}(t, q)+v \cdot \nabla f(t, q) \leq 0\right\} & \text { si } \begin{array}{c}
f(t, q) \geq 0 \\
\mathbf{R}^{3} \quad \text { sinon. }
\end{array} \\
&
\end{array}\right.
$$

Comme on l'a dit, l'équation différentielle de la Dynamique est, dans cette Section, entendue au sens propre, postulant l'absolue continuité de la fonction 
$u$ (on exclut l'éventualité de collisions). Donc $u\left(t_{1}\right)=u^{-}\left(t_{1}\right)=u^{+}\left(t_{1}\right)$, élément qui, si $f\left(t_{1}, q\left(t_{1}\right)\right)=0$, doit appartenir à la fois à $W\left(t_{1}, q\left(t_{1}\right)\right)$ et à l'ensemble défini symétriquement en remplaçant $\geq$ par $\leq$. C'est dire que $u\left(t_{1}\right)$ appartient dans ce cas au plan frontière du demi-espace fermé $W\left(t_{1}, q\left(t_{1}\right)\right)$. Le cône normal au point $u\left(t_{1}\right)$ à ce demi-espace est la demi-droite engendrée par $\nabla f\left(t_{1}, q\left(t_{1}\right)\right)$; on a vu que cette demi-droite est aussi le cône normal au point $q\left(t_{1}\right)$ à $\Phi\left(t_{1}\right)$. Par ailleurs, si $f\left(t_{1}, q\left(t_{1}\right)\right)<0$, ces deux cônes sont trivialement égaux au singleton $\{0\}$.

L'ínclusion (8) implique donc

$$
X(t, q)-m \frac{d u}{d t} \in \mathrm{N}_{W(t, q)}(u) .
$$

Si $q \in \Phi(t)$, on vérifie immédiatement que $\mathrm{N}_{W(t, q)}$, évalué en n'importe quel point $u$, est contenu dans $\mathrm{N}_{\Phi(t)}(q)$. Pourvu que $q(t) \in \Phi(t)$ soit assuré, l'inclusion (9) implique donc réciproquement l'inclusion (8).

Or (9) garantit $u \in W(t, q)$, sans quoi le second membre serait vide et il est facile d'établir [16][20]:

LEMME DE VIABILITÉ. Soit u, Lebesgue-intégrable sur un intervalle I d'origine $t_{0}$ et soit $q$ construit par (1). Si $q\left(t_{0}\right) \in \Phi\left(t_{0}\right)$ et si pour presque tout $t \in I$ on a $u(t) \in W(t, q(t))$, alors $q(t) \in \Phi(t)$ pour tout $t \in I$.

Bref, moyennant la spécification initiale $q\left(t_{0}\right) \in \Phi\left(t_{0}\right)$, (9) est équivalente à (8). Observer que ce Lemme s'attache à l'orientation du temps: si $f(t, q) \leq 0$, l'ensemble $W(t, q)$ est constitué des valeurs de la vitesse à droite compatibles avec le maintien ultérieur de cette condition.

REMARque. - Le terme "viabilité" est employé plus généralement pour désigner l'obligation faite à la trajectoire d'un processus de demeurer dans un ensemble donné. On pourra trouver dans [3] un exposé de ce sujet, développé d'ailleurs dans un contexte topologique trop raffiné pour qu'un énoncé aussi élémentaire que le Lemme ci-dessus y trouve place.

\subsection{Un exemple primitif d'algorithme $C D$}

Soit $\left[t_{\mathrm{i}}, t_{\mathrm{f}}\right]$, de longueur $h$, un pas de temps ('i' comme initial, 'f' comme final). Le pas antécédent ayant livré des valeurs $u_{\mathrm{i}}$ and $q_{\mathrm{i}}$ pour les fonctions $u$ et $q$ à $t=t_{\mathrm{i}}$, l'objectif est d'évaluer $u_{\mathrm{f}}$ et $q_{\mathrm{f}}$. On adopte $q_{\mathrm{m}}:=q_{\mathrm{i}}+\frac{1}{2} h u_{\mathrm{i}}$ comme estimation de la position à l'instant milieu $t_{\mathrm{m}}:=t_{\mathrm{i}}+\frac{1}{2} h$ et on discrétise (9) sous une forme implicite relativement à l'inconnue $u_{\mathrm{f}}$

$$
X\left(t_{\mathrm{m}}, q_{\mathrm{m}}\right)-\frac{m}{h}\left(u_{\mathrm{f}}-u_{\mathrm{i}}\right) \in \mathrm{N}_{W\left(t_{\mathrm{m}}, q_{\mathrm{m}}\right)}\left(u_{\mathrm{f}}\right) .
$$

Cette inclusion, reécrite

$$
u_{\mathrm{i}}+\frac{h}{m} X\left(t_{\mathrm{m}}, q_{\mathrm{m}}\right)-u_{\mathrm{f}} \in \mathrm{N}_{W\left(t_{\mathrm{m}}, q_{\mathrm{m}}\right)}\left(u_{\mathrm{f}}\right),
$$


(on omet le facteur strictement positif $\frac{h}{m}$ au second membre, puisque ce second membre est un cône) caractérise classiquement $u_{\mathrm{f}}$ comme point proximal de l'élément connu $u_{\mathrm{i}}+\frac{h}{m} X\left(t_{\mathrm{m}}, q_{\mathrm{m}}\right)$ dans l'ensemble convexe fermé $W\left(t_{\mathrm{m}}, q_{\mathrm{m}}\right)$

$$
u_{\mathrm{f}}=\operatorname{prox}\left(W\left(t_{\mathrm{m}}, q_{\mathrm{m}}\right), u_{\mathrm{i}}+\frac{h}{m} X\left(t_{\mathrm{m}}, q_{\mathrm{m}}\right)\right),
$$

de calcul immédiat dans le cas présent où $W$ est soit un demi-espace, soit l'espace entier.

On termine le pas par

$$
q_{\mathrm{f}}=q_{\mathrm{m}}+\frac{h}{2} u_{\mathrm{f}}
$$

Cet algorithme gère automatiquement la cessation possible du contact: c'est ce qui arrive si $u_{\mathrm{i}}+\frac{h}{m} X\left(t_{\mathrm{m}}, q_{\mathrm{m}}\right)$ tombe dans l'intérieur de $W\left(t_{\mathrm{m}}, q_{\mathrm{m}}\right)$.

Le maintien de la condition d'impénétrabilité (3) est également assuré, pourvu que cette dernière soit satisfaite à l'instant initial. Pour le problème théorique, on a vu que cela résulte du Lemme de Viabilité. Ici, le même argument ne se retrouve qu'à travers la discrétisation et on pourrait craindre, de pas en pas, une accumulation de violations de la condition (3). En pratique, si $h$ n'est pas trop grand, on constate un effet d'autocorrection évitant cette accumulation.

La précision de l'algorithme ci-dessus, de type implicite par rapport à $u$, doit beaucoup à ce que, alors que les positions $q$ sont évaluées aux extrémités de l'intervalle $\left[t_{\mathrm{i}}, t_{\mathrm{f}}\right]$, le traitement de $u$ s'effectue à mi-pas.

Il se trouve aussi que, à sa manière, l'algorithme gère les collisions éventuelles. Si $f\left(t_{\mathrm{m}}, q_{\mathrm{m}}\right)<0$, l'ensemble $W\left(t_{\mathrm{m}}, q_{\mathrm{m}}\right)$ égale $\mathbf{R}^{3}$ entier et (11) reflète simplement la dynamique d'un point libre soumis au champ $X$. S'il n'en est plus de même au pas suivant, (11) se charge de mettre $u_{\mathrm{f}}$ en conformité avec la liaison d'impénétrabilité. Cette nouvelle vitesse appartient au demi-plan frontière de $W\left(t_{\mathrm{m}}, q_{\mathrm{m}}\right)$, c'est-à-dire que la collision est traitée comme de restitution nulle [14]. On reviendra plus loin sur un traitement plus général des restitutions.

Noter que, à la différence d'une approche du problème du mouvement d'un point dans une surface par les méthodes traditionnelles de la dynamique, l'algorithme n'invoque pas la courbure de la paroi. Cette courbure se manifeste implicitement par le fait que, d'un pas au suivant, $\nabla f$ change de direction.

\section{Systèmes à contacts multiples}

\subsection{Cas sans frottement}

Au lieu de la simple inégalité (3), la dynamique d'une collection de corps met aux prises avec un systèmes d'inégalités exprimant leurs impénétrabilités mutuelles, avec même la possibilité de plusieurs contacts pour chaque paire de corps. 
Déja pour une simple particule confinée comme ci-dessus dans une région de $\mathbf{R}^{3}$, la paroi peut se composer de $\kappa$ parties régulières, éventuellement mobiles, imposant respectivement des inégalités de la forme

$$
f_{\alpha}(t, q) \leq 0, \quad \alpha=1,2, \ldots, \kappa .
$$

$\mathrm{Si}$, par exemple, les parties $f_{1}=0$ et $f_{2}=0$ de cette paroi se rejoignent selon une arête anguleuse, une position $q$ appartenant à cette arête est un point singulier de la frontière de la région $\Phi(t)$. En ce cas $\mathrm{N}_{\Phi(t)}(q)$ est, par définition, le cône convexe engendré par les deux vecteurs $\nabla f_{1}(t, q)$ et $\nabla f_{2}(t, q)$, c'est-àdire l'ensemble de leurs combinaisons linéaires à coefficients $\geq 0$. Si le contact avec chacune des deux portions de surface est sans frottement, l'ensemble des valeur possibles pour la réaction totale égale donc $-\mathrm{N}_{\Phi(t)}(q)$.

De façon générale, pour tout $t$ et tout $q$, notons

$$
J(t, q):=\left\{\alpha \in\{1, \ldots, \kappa\} \mid f_{\alpha}(t, q) \geq 0\right\} .
$$

Si $q \in \Phi(t)$, c'est l'ensemble des valeurs de $\alpha$ telles que (12) soit vérifiée comme égalité, c'est-à-dire le sous-ensemble de $\{1, \ldots, \kappa\}$ correspondant à des portions de parois que le point touche. Et $\mathrm{N}_{\Phi(t)}(q)$ se définit comme le cône convexe engendré par les $\nabla f_{\alpha}(t, q)$ pour $\alpha \in J(t, q)$ (selon une logique usuelle en Théorie de la Convexité, ce cône convexe engendré consiste dans le singleton $\{0\}$ si $J(t, q)$ est vide). Moyennant quoi, l'équation de la Dynamique du point confiné par ce système de parois s'écrit finalement sous la même forme qu'en (8).

On généralise la définition posée plus haut pour $W$ en

$$
W(t, q):=\left\{v \in \mathbf{R}^{3} \mid \forall \alpha \in J(t, q): \frac{\partial f_{\alpha}}{\partial t}+v \cdot \nabla f_{\alpha} \leq 0\right\} .
$$

(ensemble égal à $\mathbf{R}^{3}$ tout entier si $J(t, q)$ est vide), définition valable même pour des positions impliquant une violation de (12). Un raisonnement un peu plus technique que précédemment permet à nouveau, moyennant l'hypothèse $q\left(t_{0}\right) \in \Phi\left(t_{0}\right)$, de mettre l'équation de la Dynamique du point sous la forme équivalente (9).

En résumé, la description des circonstances mécanique du contact sans frottement qui, dans l'écriture (7) se présentait comme une relation entre force de contact et position, est remplacée par

$$
-r \in \mathrm{N}_{W(t, q)}(u)
$$

relation entre force, position et vitesse. On a vu en 2.3 l'intérêt algorithmique d'une tel détour. En outre, un avantage décisif de l'introduction de la vitesse est de fournir un cadre apte à prendre aussi en compte le frottement.

\subsection{Lois de contact}

Dans le cas d'une collection de corps, la non-pénétration s'exprime par des inégalités du type (12) concernant la configuration $q:=\left(q^{1}, q^{2}, \ldots, q^{n}\right) \mathrm{du}$ 
système. L'éventualité $f_{\alpha}(t, q)=0$ correspond à un contact affectant deux corps qu'on va noter $\mathcal{B}_{\alpha}$ (toujours un membre du système) et $\mathcal{B}_{\alpha}^{\prime}$ (un autre membre du système ou un obstacle extérieur de mouvement imposé). Le contact est supposé localisé en un point isolé, soit $M_{\alpha}$, mais l'éventualité d'autres contacts ponctuels entre ces deux mêmes corps n'est pas exclue.

Soit $\mathcal{U}_{\alpha}$ la vitesse relative de $\mathcal{B}_{\alpha}$ par rapport à $\mathcal{B}_{\alpha}^{\prime}$ au point $M_{\alpha}$ et soit $\mathcal{R}^{\alpha}$ la force de contact exercée par $\mathcal{B}_{\alpha}^{\prime} \operatorname{sur} \mathcal{B}_{\alpha}$. Nous appellerons loi de contact une relation édictée entre ces deux vecteurs, pouvant en outre dépendre du temps et de la configuration atteinte :

$$
\operatorname{loi}_{\alpha}\left(t, q, \mathcal{U}_{\alpha}, \mathcal{R}^{\alpha}\right)=\text { vrai. }
$$

L'écriture des équations de la Dynamique dans le paramétrage $\left(q^{1}, q^{2}, \ldots, q^{n}\right)$ met en oeuvre des composantes généralisées qu'il faut savoir relier aux vecteurs $\mathcal{U}_{\alpha}, \mathcal{R}^{\alpha}$ de l'espace physique, associés au contact étiqueté $\alpha$.

$\mathrm{Au}$ stade présent, le $n$-vecteur des vitesses $u:=\left(u^{1}, u^{2}, \ldots, u^{n}\right)$ est simplement constitué par les dérivées des fonctions $t \mapsto q^{i}$, à tout instant où ces dérivées existent. Par la construction même du paramétrage, on sait exprimer à partir de ces dérivées le vecteur $\mathcal{U}_{\alpha}$ sous la forme

$$
\mathcal{U}_{\alpha}=G_{\alpha} u+\mathcal{W}_{\alpha},
$$

où $G_{\alpha}$ est une application linéaire de $\mathbf{R}^{n}$ dans $\mathbf{R}^{3}$ et où $\mathcal{W}_{\alpha} \in \mathbf{R}^{3}$ rend compte du mouvement éventuellement imposé à $\mathcal{B}_{\alpha}^{\prime}$; ces éléments dépendent a priori de $t$ et de $q$.

Pour tout $(t, q)$, au moins dans un voisinage de la situation de contact considérée, on peut exprimer aussi en fonction de ces variables l'interstice $g_{\alpha}(t, q)$ entre $\mathcal{B}_{\alpha}$ et $\mathcal{B}_{\alpha}^{\prime}$ (angl. gap), compté selon une normale commune aux corps en regard et traité comme négatif en cas d'interpénétration. Classiquement, si dans la situation de contact en cause, on note $\mathbf{n}^{\alpha}$ un vecteur unité normal au contact, dirigé de $\mathcal{B}_{\alpha}^{\prime}$ vers $\mathcal{B}_{\alpha}$, on a pour tout mouvement différentiable $t \mapsto q(t)$ imaginé traverser cette situation

$$
\frac{d}{d t} g_{\alpha}(t, q(t))=\mathbf{n}^{\alpha} \cdot \mathcal{U}_{\alpha}, \quad \text { autrement dit } \quad \mathbf{n}^{\alpha} \cdot\left(G_{\alpha} u+\mathcal{W}_{\alpha}\right),
$$

formule qui vaut aussi plus précisément pour des $\frac{d}{d t}$ et des vitesses $\grave{a}$ droite ou à gauche de l'instant en cause. Les éléments $\mathbf{n}^{\alpha}, G_{\alpha}, \mathcal{W}_{\alpha}$ sont ici des fonctions connues de $t$ et $q$, fonctions dont la définition est supposée étendue conventionnellement à un voisinage de la situation de contact en question.

Pour les besoins du calcul, on imaginera la loi (16) prolongée elle aussi à un voisinage de la situation de contact et on supposera qu'il s'agit proprement d'une loi de contact, c'est-à-dire

$$
g_{\alpha}(t, q)>0 \Rightarrow \mathcal{R}^{\alpha}=0 .
$$

La loi (16) sera dite de type prospectif si elle est constituée de manière à assurer les implications 


$$
\begin{gathered}
g_{\alpha}(t, q) \leq 0 \Rightarrow \mathbf{n}^{\alpha} \cdot \mathcal{U}_{\alpha} \geq 0 \\
\mathbf{n}^{\alpha} \cdot \mathcal{U}_{\alpha}>0 \Rightarrow \mathcal{R}^{\alpha}=0 .
\end{gathered}
$$

L'importance de l'implication (20) est la suivante. Si la fonction $f$ du parag. 2.2 est prise égale à $-g_{\alpha}$, l'identité (18) nous permet d'identifier dans le contexte présent l'ensemble $W$ et d'appliquer le Lemme de Viabilité. On conclut que, si le contact étiqueté $\alpha$ est régi par une loi possédant cette propriété et si aucune interpénétration des corps en présence n'a lieu à un instant initial, il en sera de même dans la suite.

L'implication (21) justifie le qualificatif "prospectif". Dans le contexte de Dynamique Non Régulière qui est celui du présent article, on doit être prêt à rencontrer des discontinuités temporelles. L'idée sous-jacente à l'écriture (21) est que la loi formulée ne concerne pas proprement les valeurs actuelles des fonctions $\mathcal{U}_{\alpha}$ et $\mathcal{R}^{\alpha}$, mais leurs limites à droite. La limite à droite de $\mathbf{n}^{\alpha} . \mathcal{U}_{\alpha}$ égale la dérivée à droite de la fonction $t \mapsto g_{\alpha}(t, q(t))$. Si cette dérivée est $>0$, le contact cesse et $\mathcal{R}^{\alpha}$ devient nul; donc sa limite à droite est nulle.

On comprendra mieux l'importance du concept de loi de type prospectif lorsqu'on en viendra, au parag. 6.1 à l'écriture d'un algorithme à temps discrétisé de type implicite pour calculer l'évolution.

L'écriture de l'équation de la dynamique du système, demande que chacune des forces que le système subit soit représentée par ses composantes généralisées, formant un élément de $\mathbf{R}^{n}$. En particulier, notons $r^{\alpha}$ l'élément de $\mathbf{R}^{n}$ constitué par les composantes généralisées des forces de contact au point $M_{\alpha}$. Selon la machinerie classique de la Mécanique Analytique,

$$
r^{\alpha}=G_{\alpha}^{*} \mathcal{R}^{\alpha},
$$

où l'application linéaire $G_{\alpha}^{*}$ de $\mathbf{R}^{3}$ dans $\mathbf{R}^{n}$ est la transposée de $G_{\alpha}$. Dans le cas où les corps $\mathcal{B}_{\alpha}$ et $\mathcal{B}_{\alpha}^{\prime}$ sont tous les deux des membres du système, cette expression englobe automatiquement les deux forces $\mathcal{R}^{\alpha}$, exercée par $\mathcal{B}_{\alpha}^{\prime}$ sur $\mathcal{B}_{\alpha}$ et la force antagoniste $-\mathcal{R}^{\alpha}$ exercée par $\mathcal{B}_{\alpha}$ sur $\mathcal{B}_{\alpha}^{\prime}$. En revanche si, comme on l'a envisagé plus haut, $\mathcal{B}_{\alpha}^{\prime}$ est un obstacle extérieur, seule la force $\mathcal{R}^{\alpha}$ est subie par le système et non son antagoniste, mais la formule (22) reste vraie pour exprimer les composantes généralisées de cette seule force.

\subsection{Equations de la Dynamique régulière}

Si on postule comme précédemment un mouvement assez régulier pour que la fonction vitesse soit absolument continue, la dynamique du système est régie par une équation de la forme suivante, dont les deux membres sont des éléments de $\mathbf{R}^{n}$

$$
A(t, q) \frac{d u}{d t}=F(t, q, u)+\sum_{\alpha} r^{\alpha} .
$$

La matrice symétrique $n \times n$ notée $A$ (matrice d'inertie) est définie positive si le paramétrage est régulier. Dans la fonction connue $F$ sont agglomérées 
les composantes généralisées de forces données, dépendant de façon régulière du temps, de la position et de la vitesse, ainsi que des termes dynamiques ne contenant pas la dérivée $\frac{d u}{d t}$ (il est parfois suggestif de les classer en termes "gyroscopiques" et en termes "centrifuges").

Les $r^{\alpha}$ sont liés par (22) aux forces de contact $\mathcal{R}^{\alpha}$, lesquelles sont ellesmêmes reliées aux vitesses relative locales $\mathcal{U}_{\alpha}$ par des lois de contact de la forme (16). Si ces lois de contact sont énoncées de manière à fournir $\mathcal{R}^{\alpha}=0$ lorsque le contact $\alpha$ n'est pas effectif, il sera indifférent de restreindre $\alpha$ à l'ensemble $J(t, q)$ ou à lui laisser parcourir la totalité de la liste $\{1,2, \ldots, \kappa\}$.

Les $\mathcal{U}_{\alpha}$ sont liés à $u$ par (17).

Enfin on doit rappeler que les fonctions $q$ et $u$ sont liées par (1).

GÉNÉRALisATion. - Il est souvent avantageux d'exprimer la Dynamique par une équation de la même forme que (23) mais concernant une fonction vitesse $t \mapsto u \in \mathbf{R}^{n}$ liée à la fonction position $t \mapsto q$ par une relation cinématique plus compliquée que (1). De fait, dans l'étude de l'évolution d'un corps rigide, il est usuel de faire figurer comme composantes de $u$, au lieu des dérivées de certains paramètres angulaires apparaissant dans $q$, les composantes du vecteur vitesse angulaire du corps, relativement à des axes principaux centraux d'inertie qui lui sont attachés. L'avantage est que les termes correspondants dans la matrice de masse $A$ ci-dessus sont indépendants de $(t, q)$ et nuls en dehors de la diagonale. Le prix à payer est que (1) devra être remplacée par une intégration de formules de cinématique élémentaire, numériquement facile.

Définir $u$ de cette manière n'empèche pas d'exprimer les vitesses relatives locales $\mathcal{U}_{\alpha}$ sous la forme (17), auquel cas (22) continue de fournir les $r^{\alpha}$ à insérer dans (23).

\section{Frottement de Coulomb}

\subsection{Contact frottant}

Le contact avec frottement sec entre corps déformables, discrétisés en éléments finis, fait depuis un certain nombre d'années l'objet de publications [2][8][11], prenant en compte éventuellement aussi une adhésion [24]. Bien que ces travaux aient le plus souvent été limités à des évolutions quasi-statiques, ils permettent d'apprécier la diversité des procédures possibles pour appréhender à la fois le caractère unilatéral de la liaison d'impénétrabilité et la présence de seuils dans les lois de frottement sec.

Une des sources de l'intérêt du remplacement de (7) par (??) est de conduire à des calculs invoquant des ensembles convexes dans l'espace des vitesses éventuelles du système, lequel est un espace vectoriel, alors que, dans la variété des configurations, la notion de convexité, utile dans diverses techniques numériques, n'a pas de sens. De plus, ce type de formulation en termes de vitesses locales se prète directement à la prise en compte de frottements, régis par des lois empiriques. 
Il est facile d'énoncer la loi de Coulomb en une liste de conditions constituant une loi de contact de type prospectif. L'algorithme résultant gèrera automatiquement la non-interpénétration. Au plan théorique, on peut trouver plus convaincant d'appliquer à la loi de Coulomb le formalisme des bi-potentiels [8]. Ce formalisme engendre automatiquement une loi de contact de type prospectif et, lorqu'on arrive à la construction d'un algorithme, il remplace la formule de proximation (11) par une caractérisation qui est encore de type extrémal (voir aussi [15]).

Le cas particulier très simple traité dans ce paragraphe permet également de constater l'aptitude de l'algorithme $C D$ à tester les équilibres. Si, à l'instant $t_{\mathrm{i}}$, le point $q_{\mathrm{i}}$ est, compte tenu d'un frottement de Coulomb, une position d'équilibre, l'algorithme lancé avec $u_{\mathrm{i}}=0$ fournit $u_{\mathrm{f}}=0$ et $q_{\mathrm{f}}=q_{\mathrm{i}}$.

\section{Collisions}

\subsection{Dynamique percussionnelle}

On a vu dans le cas spécial du parag. 2.3 comment un algorithme $C D$ peut faire face formellement à une collision. La difficulté ne se situe pas dans l'occurence d'un saut de vitesse, mais dans la nécessité de posséder des lois phénoménologiques maniables résumant avec assez de précision la complexité d'interactions brèves mettant en jeu de grandes forces.

Il est usuel d'admettre que le phénomène se déroule sur un intervalle de temps $\left[t_{c}, t_{c}+\tau\right]$ suffisamment court pour que les déplacements y soient négligeables. La matrice $A$ de l'équation (23) est ainsi traitée comme constante sur cette durée ; on intègre les deux membres sur l'intervalle. Le terme $F$ donne une contribution négligeable, mais non les forces de contact, pour lesquelles on attend des valeurs d'autant plus grandes que l'interaction est plus brève. Il reste

$$
u\left(t_{c}+\tau\right)-u\left(t_{c}\right)=A^{-1} \sum_{\alpha} s^{\alpha} .
$$

Les $s^{\alpha}$ sont les intégrales des $r^{\alpha}$; ces dernières quantités sont reliées aux forces de contact $\mathcal{R}^{\alpha}$ par (22) et, comme avec $A$, on suppose la durée $\tau$ suffisamment courte pour que $G_{\alpha}^{*}$ soit traitée comme une matrice constante. Celà donne

$$
s^{\alpha}=G_{\alpha}^{*} \mathcal{S}^{\alpha},
$$

où $\mathcal{S}^{\alpha}$, intégrale de $\mathcal{R}^{\alpha}$ sur l'intervalle, est la percussion de contact. Noter que les valeurs de $\alpha$ à considérer ne se limitent pas nécessairement à l'étiquette du contact brusquement introduit par la collision : si les corps qui se rencontrent font partie d'amas ayant déja des contacts, des percussions doivent aussi être attendues en ces contacts.

A ce stade de la formulation, la durée $\tau$ est oubliée et le premier membre de $(24)$ peut aussi bien s'interpréter comme $u^{+}\left(t_{c}\right)-u^{-}\left(t_{c}\right)$, amplitude d'un saut instantané de la fonction $u$. 
Il manque des informations sur $\mathcal{S}^{\alpha}$, pour qu'on puisse déterminer $u^{+}\left(t_{c}\right)$, lequel servira de vitesse initiale lorsqu'on calculera le mouvement consécutif à la collision. Depuis Darboux, il est traditionnel de procéder à une analyse du processus de collision grâce à un changement d'échelle, indexant l'évolution des vitesses par un micro-temps relativement auquel on invoque les équations de la Dynamique régulière sans pour autant que les positions changent. Les procédures de cette sorte comportent diverses variantes, un point délicat étant de discuter l'importance éventuelle de déformations microscopiques des corps au voisinage des lieux d'impact. Elles révèlent au moins que la vitesses relative $\mathcal{U}_{\alpha}$ ne conserve pas en général une direction fixe durant lévolution microscopique. Les lois de contact, telles que la loi de Coulomb formulée à la section précédente, sont essentiellent des relations non-linéaires entre $\mathcal{U}_{\alpha}$ et $\mathcal{R}^{\alpha}$. Les opérations d'intégration par lesquelles on définirait la moyenne de $\mathcal{U}_{\alpha}$ et la percussion $\mathcal{S}^{\alpha}$ ne commutent pas en général avec de telles relations (toutefois, la loi de Coulomb étant positivement homogène de degré 1 par rapport à $\mathcal{U}_{\alpha}$ et à $\mathcal{R}^{\alpha}$, la commutation se justifie si on a des raisons d'affirmer que $\mathcal{U}_{\alpha}$ conserve une direction et un sens fixes durant le processus).

\subsection{Vitesses pondérées et restitution}

Les techniques numériques auxquelles le présent article sert d'introduction visent la dynamique de granulats ou de maçonneries pouvant comporter plusieurs dizaines de milliers de contacts. Si une collision survient, il semble hors de portée d'analyser les micro-évolutions induites en chacun de ces contacts. Ce qui suit réussit à adapter à de telles situations l'approche pragmatique traditionnelle grâce à laquelle seules les vitesses avant choc (connues) et les vitesses après choc (les inconnues du problème) entrent dans les formules.

On va admettre qu'en chaque contact - soit $\alpha$ son étiquette - une loi de contact telle que la loi de Coulomb (positivement homogène de degré 1 et de type prospectif) relie la percussion $\mathcal{S}^{\alpha}$ à une vitesse locale moyenne $\mathcal{U}_{\alpha}^{a}$ ("a" comme average). Celle-ci est définie par une pondération entre les valeurs avant et après choc $\mathcal{U}_{\alpha}^{-}$et $\mathcal{U}_{\alpha}^{+}$, pondération qui peut d'ailleurs invoquer des coefficients différents pour les composantes normales $\mathcal{U}_{\alpha N}$ et tangentielles $\mathcal{U}_{\alpha T}$ :

$$
\begin{aligned}
& \mathcal{U}_{\alpha N}^{a}=\frac{\rho_{\alpha}}{1+\rho_{\alpha}} \mathcal{U}_{\alpha N}^{-}+\frac{1}{1+\rho_{\alpha}} \mathcal{U}_{\alpha N}^{+} \\
& \mathcal{U}_{\alpha T}^{a}=\frac{\tau_{\alpha}}{1+\tau_{\alpha}} \mathcal{U}_{\alpha T}^{-}+\frac{1}{1+\tau_{\alpha}} \mathcal{U}_{\alpha T}^{+} .
\end{aligned}
$$

Les paramètres empiriques $\rho_{\alpha}$ et $\tau_{\alpha}$ seront appelés coefficient de restitution normale et coefficient de restitution tangentielle, dénominations justifiées par ce qui suit.

La loi de contact invoquée étant de type prospectif, les implications (20) et (21) montrent que la percussion $\mathcal{S}^{\alpha}$ ne peut différer de zéro que si $\mathcal{U}_{\alpha N}^{a}=0$, c'est-à-dire $\mathcal{U}_{\alpha N}^{+}=-\rho_{\alpha} \mathcal{U}_{\alpha N}^{-}$, ce qui est formellement l'équation de restitution 
de Newton. Mais la procédure présente est plus riche qu'une simple loi de restitution, en ce qu'elle permet aussi $\mathcal{S}^{\alpha}=0$ conjointement à $\mathcal{U}_{\alpha N}^{a}>0$. C'est le calcul global, invoquant tous les contacts par l'intermédiaire de (24), qui tranche entre ces deux alternatives.

De manière analogue, ce calcul global peut, si le frottement est suffisant, déboucher sur le cas "vitesse tangentielle nulle" de la loi de Coulomb. Alors $\mathcal{U}_{\alpha T}^{+}=-\tau_{\alpha} \mathcal{U}_{\alpha T}^{-}$, ce qui exprime une restitution tangentielle.

L'exemple familier du balancement par lequel un bloc rectangulaire élancé, posé sur un plan horizontal, atteint finalement son équilibre après des alternances de pivotement autour des deux coins inférieurs, montre l'inaptitude de la formule de restitution de Newton à modéliser les collisions dans un système exhibant plusieurs contacts : lorsque, à l'issue d'un pivotement autour de l'un des coins, l'autre coin vient heurter le plan horizontal, cette formule ne permettrait pas au premier point de décoller. En revanche, la technique des vitesses pondérées, même avec des coefficients de restitution nuls, fournit des résultats plausibles.

Déja dans le cas le plus simple, celui de la collision entre deux corps par ailleurs libres, les formules traditionnelles invoquant un coefficient de restitution (qu'il soit de Newton, de Poisson ou d'autres) n'ont guère de portée pratique hors du cas des corps sphériques [26]. Si une collision affecte des objets faisant par ailleurs partie d'amas de corps déja en contact, des percussions de liaison doivent être attendues en tous les contacts existants, situation encore plus difficile à analyser.

Pragmatiquement, l'implémentation la plus usuelle de la méthode $C D$ traite les collisions comme suit.

Si (16) consiste dans la loi de Coulomb, cette relation est positivement homogène de degré 1 par rapport aux variables $\mathcal{U}_{\alpha}$ et $\mathcal{R}^{\alpha}$. Cela permet de conduire le pas de calcul en traitant $\mathcal{R}^{\alpha}$ non pas comme la force de contact, mais comme l'impulsion totale transmise par le contact sur l'intervalle $\left[t_{\mathrm{i}}, t_{\mathrm{f}}\right]$. Le parti adopté ci-dessus de construire une discrétisation de type implicite a conduit à porter dans la loi de contact la valeur (inconnue) $\mathcal{U}_{\alpha}^{\mathrm{f}}$ de la vitesse locale en fin de pas. On a vu dans le cas spécial du Parag. 2.3 qu'une collision éventuelle se trouve alors automatiquement traitée comme inélastique. Si, au lieu de cela, on porte dans la loi de contact la demi-somme de $\mathcal{U}_{\alpha}^{\mathrm{f}}$ et de la valeur (connue) $\mathcal{U}_{\alpha}^{\mathrm{i}}$ de la vitesse locale en début de pas, on obtient un choc élastique. De façon générale, en portant dans la loi de contact une moyenne pondérée de $\mathcal{U}_{\alpha}^{\mathrm{i}}$ et $\mathcal{U}_{\alpha}^{\mathrm{f}}$ on créé une loi de choc impliquant frottement et restitution (en appliquant des pondérations différentes aux composantes normales et tangentielles, on peut même distinguer une restitution normale et une restitution tangentielle). L'avantage principal de ce traitement est sa cohérence dans le cas où de multiples contacts sont impliqués dans une collision, mais il ne faudrait pas y voir la proposition d'une théorie générale des chocs. 


\subsection{Equations de la Dynamique Non Régulière}

On recherche le mouvement du système sur un intervalle de temps $I$, d'origine $t_{0}$. L'inconnue principale étant, comme on l'a dit, la fonction vitesse $t \mapsto u \in$ $\mathbf{R}^{n}$, nous nous limitons pour abréger au cas où la fonction position $t \mapsto q \in \mathbf{R}^{n}$ s'en déduit par l'intégration (1).

Même si la fonction $u$ est construite de façon moins directe (cf. parag. 3.3), on peut finalement généraliser l'équation (23) sous la forme d'une égalité de mesures à valeurs dans $\mathbf{R}^{n}$

$$
A(t, q) d u=F(t, q, u) d t+\sum_{\alpha} s^{\alpha} .
$$

Ici, $d u$ désigne la mesure différentielle de $u$, égale à $u_{t}^{\prime} d t$ sur tout sous-intervalle de $I$ où $u$ est absolument continue, mais qui peut aussi comporter des atomes, c'est-à-dire des mesures concentrées aux instants de discontinuité éventuelle des vitesses [17]: le poids d'un tel atome est égal au saut $u^{+}-u^{-}$.

Les $s^{\alpha}$ sont les composantes généralisées des mesures impulsions $\mathcal{S}^{\alpha}$ aux divers points de contact. Sur un sous-intervalle où le mouvement est régulier, on a $\mathcal{S}^{\alpha}=\mathcal{R}^{\alpha} d t$ et $s^{\alpha}=r^{\alpha} d t$, ce qui permet d'invoquer (22) pour chaque $t$. En revanche, sur la durée "infiniment courte" d'une collision, $G_{\alpha}^{*}(t, q)$ est traité comme constant : par intégration sur cette durée, il vient donc une relation de même forme entre $s^{\alpha}$ et $\mathcal{S}^{\alpha}$. Au total, sur l'intervalle $I$ entier on a la relation suivante entre mesures, qu'elles aient ou non une densité par rapport à $d t$,

$$
s^{\alpha}=G_{\alpha}^{*} \mathcal{S}^{\alpha},
$$

écriture légitime puisque $t \mapsto G_{\alpha}^{*}(t, q(t))$ est continue.

\section{Implémentation}

\subsection{Discrétisation}

Si on évite les instants où la matrice $A$ deviendrait singulière, la fonction matricielle $t \mapsto A^{-1}(t, q(t))$ est continue. En multipliant par cette fonction les deux membres de (28) (et en renommant l'indice de sommation), on obtient

$$
d u=A^{-1} F d t+A^{-1} \sum_{\beta} s^{\beta} .
$$

Comme au parag.2.3, soit $\left[t_{\mathrm{i}}, t_{\mathrm{f}}\right]$, de longueur $h$, un pas de temps ; on pose $t_{\mathrm{m}}:=t_{\mathrm{i}}+\frac{1}{2} h$ et $q_{\mathrm{m}}:=q_{\mathrm{i}}+\frac{1}{2} h u_{\mathrm{i}}$. Vu leur continuité, les matrices $A^{-1}, G_{\beta}, G_{\beta}^{*}$ seront traitées sur tout l'intervalle comme conservant les valeurs qu'elles ont au point $\left(t_{\mathrm{m}}, q_{\mathrm{m}}\right)$, tandis qu'on adoptera pour $F$ la valeur constante $F\left(t_{\mathrm{m}}, q_{\mathrm{m}}, u_{\mathrm{i}}\right)$. Moyennant quoi, en intégrant les deux membres de (30) sur l'intervalle, il vient

$$
u_{\mathrm{f}}=u_{\mathrm{i}}+A^{-1} F h+\sum_{\beta} A^{-1} G_{\beta}^{*} \mathcal{S}^{\beta},
$$


où $\mathcal{S}^{\beta}$, intégrale de $\mathcal{R}^{\beta}$, constitue l'impulsion de contact au point $M_{\beta}$ pour le pas concerné. Une telle discrétisation résumant un bilan d'impulsions sur l'intervalle $\left[t_{\mathrm{i}}, t_{\mathrm{f}}\right]$, ne postule pas l'existence des accélérations : elle vaut aussi bien si l'intervalle comporte des collisions. Le cas d'une collision survenant exactement à l'une des extrémités de l'intervalle n'est pas exclu : à cet égard, on peut dire que les valeurs indexées 'i' simulent des limites à gauche et les valeurs indexées 'f $\mathrm{f}$ ' des limites à droite.

L'indice de sommation n'a besoin de parcourir que l'ensemble des étiquettes des contacts actifs. Avec la notation (13), on adopte $J_{\mathrm{m}}:=J\left(t_{\mathrm{m}}, q_{\mathrm{m}}\right)$ comme estimation de cet ensemble pour toute la durée du pas. Pour chaque $\alpha \in J_{\mathrm{m}}$, on doit disposer d'une loi de contact liant $\mathcal{S}^{\alpha}$ aux variables cinématiques. On va admettre que la vitesse relative des corps en contact au point $M_{\alpha}$ intervient sous la forme d'une moyenne pondérée comme en (26) et (27), à ceci près que les rôles tenus alors par les limites à gauche et à droite d'un instant de collision $t_{c}$ sont joués maintenant par les valeurs de $\mathcal{U}_{\alpha}$ en $t_{\mathrm{i}}$ et $t_{\mathrm{f}}$. Si on se limite à des lois du type de la loi de Coulomb, laquelle est homogène séparément par rapport aux composantes normale et tangentielle de la vitese relative locale, et si on note $G_{\alpha N}$ et $G_{\alpha T}$ la composante normale et la composante tangentielle de $G_{\alpha}$, on peut chasser les dénominateurs, d'où

$$
\begin{aligned}
\forall \alpha \in J_{\mathrm{m}}: \quad & \operatorname{loi}_{\alpha}\left(\rho_{\alpha} G_{\alpha N} u_{\mathrm{i}}+G_{\alpha N} u_{\mathrm{f}}+\left(1+\rho_{\alpha}\right) \mathcal{W}_{\alpha N},\right. \\
& \left.\tau_{\alpha} G_{\alpha T} u_{\mathrm{i}}+G_{\alpha T} u_{\mathrm{f}}+\left(1+\tau_{\alpha}\right) \mathcal{W}_{\alpha T}, \mathcal{S}^{\alpha}\right)=\text { vrai }
\end{aligned}
$$

Le problème va être de résoudre le système (31)(32) par rapport à l'inconnue $u_{\mathrm{f}}$, après quoi le pas se terminera par $q_{\mathrm{f}}=q_{\mathrm{m}}+\frac{1}{2} h u_{\mathrm{f}}$.

Remarque 1. - Traiter $F$ comme constant sur l'intervalle $\left[t_{\mathrm{i}}, t_{\mathrm{f}}\right]$ suppose que les forces "élastiques" éventuelles n'ont qu'une dépendance douce à l'égard de la position $q$. En revanche, si les élasticités sont trop raides, on peut appliquer une procédure due à M. Jean [11], consistant à remplacer $A$ par une matrice combinant inerties et coefficients d'élasticité linéaire.

REMARQue 2. - L'occurrence de collisions est révélée par un ensemble $J_{\mathrm{m}}$ contenant des contacts inexistants au pas précédent. Comme on l'a souligné dans la section précédente, les impulsions aux autres points de contact peuvent s'en trouver affectées. L'algorithme prend celà en compte, mais présente toutefois le défaut suivant.

\subsection{Procédé itératif}

L'approche la plus usuelle pour la résolution numérique du problème fortement non-linéaire (31)(32) consiste en une méthode d'itération inspirée de la technique de Gauss-Seidel.

Supposons qu'une approximation $\left(u_{\mathrm{f}}^{\text {esti }}, \mathcal{S}_{\text {esti }}^{\beta}\right), \beta$ prenant toutes les valeurs de $J_{\mathrm{m}}$, ait été obtenue, et qu'elle satisfasse exactement (31). On va en déduire une autre approximation $\left(u_{\mathrm{f}}^{\text {corr }}, \mathcal{S}_{\text {corr }}^{\beta}\right)$ en altérant seulement $\mathcal{S}^{\alpha}$, autrement dit 
$\mathcal{S}_{\text {corr }}^{\beta}=\mathcal{S}_{\text {esti }}^{\beta}$ si $\beta \neq \alpha$. On demande à cette nouvelle approximation de vérifier elle aussi (31), c'est-à-dire par différence

$$
u_{\mathrm{f}}^{\text {corr }}=u_{\mathrm{f}}^{\text {esti }}+A^{-1} G_{\alpha}^{*}\left(\mathcal{S}_{\text {corr }}^{\beta}-\mathcal{S}_{\text {esti }}^{\beta}\right)
$$

et de vérifier la loi du contact d'étiquette $\alpha$ telle qu'elle est écrite en (32). On élimine de cette dernière l'inconnue $u_{\mathrm{f}}^{\text {corr }}$ en appliquant $G_{\alpha}$ aux deux membres de (33), d'où en séparant les composantes normale et tangentielle $G_{\alpha N}, G_{\alpha T}$

$$
\begin{aligned}
\operatorname{loi}_{\alpha}\left(\mathcal{U}_{\alpha N}^{\text {const }}+G_{\alpha N} u_{\mathrm{f}}^{\text {esti }}+H_{\alpha N}\left(\mathcal{S}_{\text {corr }}^{\alpha}-\mathcal{S}_{\text {esti }}^{\alpha}\right)\right. \\
\left.\mathcal{U}_{\alpha T}^{\text {const }}+G_{\alpha T} u_{\mathrm{f}}^{\text {esti }}+H_{\alpha T}\left(\mathcal{S}_{\text {corr }}^{\alpha}-\mathcal{S}_{\text {esti }}^{\alpha}\right), \mathcal{S}_{\text {corr }}^{\alpha}\right)=\text { vrai. }
\end{aligned}
$$

Les matrices $H_{\alpha N}$ et $H_{\alpha T}$ expriment les composantes normale et tangentielle de $H_{\alpha}=G_{\alpha} A^{-1} G_{\alpha}^{*}$, application linéaire symétrique positive de $\mathbf{R}^{3}$ dans $\mathbf{R}^{3}$ que nous proposons d'appeler l' opérateur de Delassus. En quelque sorte, cet opérateur décrit l'inertie du système "telle qu'on la voit depuis le point de contact $M_{\alpha}$ ". Dans le cas usuel où $G_{\alpha}: \mathbf{R}^{n} \rightarrow \mathbf{R}^{3}$ est surjective (i.e. $G_{\alpha}^{*}: \mathbf{R}^{3} \rightarrow \mathbf{R}^{n}$ injective), l'opérateur $H_{\alpha}$ est défini positif.

Apparaissent aussi les composantes d'un élément $\mathcal{U}_{\alpha}^{\text {const }}$, ainsi noté parce que, pour chaque $\alpha$, il demeure constant au cours des iterations :

$$
\begin{aligned}
& \mathcal{U}_{\alpha N}^{\text {const }}=\rho_{\alpha} G_{\alpha N} u_{\mathrm{i}}+\left(1+\rho_{\alpha}\right) \mathcal{W}_{\alpha N} \\
& \mathcal{U}_{\alpha T}^{\text {const }}=\tau_{\alpha} G_{\alpha T} u_{\mathrm{i}}+\left(1+\tau_{\alpha}\right) \mathcal{W}_{\alpha T}
\end{aligned}
$$

La résolution de (34) est l'étape type du traitement d'un système qui présenterait un seul contact. On l'effectue de manière répétée avec $\alpha$ parcourant cycliquement $J_{\mathrm{m}}$.

Le traitement n'implique pas le stockage de grosses matrices. En effet, chacune des application linéaires $G_{\alpha}: \mathbf{R}^{n} \rightarrow \mathbf{R}^{3}$ implique au plus une paire de solides libres. La partie non nulle de la matrice correspondante est donc au plus un rectangle de taille $12 \times 3$ (et même seulement $6 \times 2$ dans les modèles bidimensionnels).

\section{Références}

[1] V. Acary \& M. Jean. Numerical simulation of monuments by the contact dynamics method, in Monument-98, Workshop on Seismic Performance of Monuments, Lisbon, 69-78, 1998.

[2] P. Alart \& A. Curnier. A mixed formulation for frictional contact problems prone to Newton like methods, Comput. Meth. Appl. Mech. Engrg., 92, 353$375,1991$.

[3] J. P. Aubin. Viability theory, Birkhäuser-Verlag, Basel/Boston, 1990.

[4] J. P. Aubin \& A. Cellina, Differential inclusions, Springer-Verlag, Berlin, 1984. 
[5] B. Brogliato. Nonsmooth mechanics. Models, dynamics and control, 2d. ed., Springer-Verlag, 1999.

[6] P. A. Cundall, A computer model for simulating progressive large scale movements of blocky rock systems, Proceedings of the Symposium of the International Society of Rock Mechanics, (Nancy, France, 1971), Vol.1, 132150 .

[7] E. Delassus, Mémoire sur la théorie des liaisons finies unilatérales, Ann. Sci. Ecole Norm. Sup., 34, 95-179, 1917.

[8] G. de Saxcé \& Z. Q. Feng. New inequation and functional for contact with friction. J. Mech. of Struct. and Machines,19, 301-325, 1991.

[9] F. Génot \& B. Brogliato. New results on Painlevé paradoxes, European Journal of Mechanics, A/Solids, 18, p. 653-677, 1999.

[10] E. J. Haug. Computer aided kinematics and dynamics, Vol. I: Basic methods, Allyn and Bacon, Boston, 1989.

[11] M. Jean. Frictional contact in collections of rigid or deformable bodies: numerical simulation of geomaterials. In A. P. S. Selvadurai \& M. J. Boulon (eds.) Mechanics of Geomaterial Interfaces. Elsevier, Amsterdam, 453-486, 1995.

[12] M. D. P. Monteiro Marques, Differential Inclusions in Nonsmooth Mechanical Problems: Shocks and Dry Friction, Birkhäuser, Basel/Boston/Berlin, 1993.

[13] J. J. Moreau. Les liaisons unilatérales et le principe de Gauss, Comptes Rendus Acad. Sci. Paris, 256, 871-874, 1963.

[14] J. J. Moreau. Standard inelastic shocks and the dynamics of unilateral constraints. In G. Del Piero \& F. Maceri (eds.), Unilateral Problems in Structural Analysis, CISM Courses and Lectures, Vol. 288, Springer-Verlag, Wien/New York, 173-221, 1985.

[15] J. J. Moreau. Une formulation du contact à frottement sec; application au calcul numérique, Comptes Rendus Acad. Sci. Paris, Sér.II, 302, 799-801, 1986.

[16] J. J. Moreau. Unilateral contact and dry friction in finite freedom dynamics. In J. J. Moreau \& P. D. Panagiotopoulos (eds.), Nonsmooth Mechanics and Applications, CISM Courses and Lectures, Vol. 302, Springer-Verlag, Wien/New York, 1-82, 1988.

[17] J. J. Moreau, Bounded variation in time. In J. J. Moreau, P. D. Panagiotopoulos \& G. Strang (eds.), Topics in Nonsmooth Mechanics, Birkhäuser, Basel/Boston/Berlin, 1-74, 1988. 
[18] J. J. Moreau. Some numerical methods in multibody dynamics : application to granular materials. Eur. J. Mech., A/Solids, 13, nº4-suppl., 93-114, 1994.

[19] J. J. Moreau. Numerical investigation of shear zones in granular materials. In P. Grassberger \& D. E. Wolf (eds.), Friction, Arching, Contact Dynamics, World Scientific, Singapore, 233-247, 1997

[20] J. J. Moreau. Some basics of unilateral dynamics. In F. Pfeiffer \& C. Glocker (eds.) Unilateral Multibody Dynamics, Kluwer, Dordrecht, 1999, à paraître.

[21] J. J. Moreau. Numerical aspects of the sweeping process. In J. A. C. Martins \& A. Klarbring (eds.), Computational Modeling of Contact and Friction, special issue of Computer Meth. in Appl. Mech. and Engng., 1999, à paraître.

[22] J. J. Moreau. Evolution en présence de liaisons unilatérales: notions de base. In D. Guédra-Degeorges, P. Ladevèze, M. Raous (eds.), Actes du 4ème Colloque National en Calcul des Structures (Giens, France, 18-21. May 1999), Teknea, Toulouse, 25-40 (ISBN 2-87717-071-3).

[23] F. Pfeiffer \& C. Glocker. Multibody Dynamics with Unilateral Contacts, John Wiley and Sons, New York, 1996.

[24] M. Raous, L. Cangemi \& M. Cocu. A consistent model coupling adhesion, friction and unilateral contact, Computer Methods in Applied Mechanics and Engineering, 177, p. 383-399, 1999.

[25] D. E. Stewart \& J. C. Trinkle. An implicit time-stepping scheme for rigid body dynamics with inelastic collisions and Coulomb friction, Int. J. Numer. Methods Eng., 39, 2673-2691, 1996.

[26] D. Stoianovici \& Y. Hurmuzlu. A critical study of the applicability of rigid body collisions theory. A.S.M.E. J. Appl. Mech., 63, 307-316, 1996.

[27] N. Strömberg, L. Johansson \& A. Klarbring. Derivation and analysis of a generalized standard model for contact friction and wear, Int. J. Solids Structures, 33, p. 1817-1836, 1996.

[28] W. J. Stronge. Unraveling paradoxical theories for rigid body collisions. A.S.M.E. J. Appl. Mech., 58, 1049-1055, 1991.

[29] O. R. Walton. Application of molecular dynamics to macroscopic particles. Int. J. Engng. Sci., 22:1097-1107, 1984. 